\title{
The importance to engage: education and outreach highlights
}

\section{Pedro Abreu ${ }^{a}$ and the IPPOG Collaboration}

a LIP and IST,

Av. Prof. Gama Pinto, 2, 1649-003, Lisboa, Portugal

E-mail: abreu@lip.pt

In this report I present some selected highlights of the contributions submitted to the Education and Outreach track of this conference, after an inspiring success story and an introduction to IPPOG and its activities, that reflect to my best knowledge what was presented at the conference.

Prague, Czech Republic (virtual meeting) 


\section{Introduction}

Let me start by thanking the organization for the great honour and invitation to contribute some thoughts related to education and outreach in Particle Physics and present some selected highlights of the contributions to the Education \& Outreach track of this conference.

Education and Outreach in Particle Physics are of utmost importance nowadays, especially when considering the size, timespan, cost and human resources required for the present experiments and future projects in Particle Physics.

This report is further structured in four sections:

2. It's all about students, presenting a rough study case based on Portuguese high-school students, of the importance to engage and the impact of outreach in particle physics;

3. It's all about Networks and Activities, presenting the international collaboration IPPOG International Particle Physics Outreach Group, and some of its activities, that engage the students and the general public into the excitement of particle physics and its benefits;

4. It's all about You, presenting a selected highlight of the contributions to the Education and Outreach track of this Conference;

5. Conclusions.

\section{It's all about Students}

Present students are tomorrow's scientists. But at the end of the last century, the decrease of the interest of secondary school students to follow courses in the STE[A]M areas was becoming a serious issue. Many plans were put forward to help regain that interest in the minds of our youth and, of particular importance, was 2005 - World/International Year of Physics with the associated many outreach activities related to physics that were proposed along the year. It was in 2005 that EP[P]OG - the European Particle Physics Outreach Group - launched the International Masterclasses in Particle Physics [1] (see next section).

But despite the many activities in 2005, from Physics Roadshows to many talks at schools and other premises, which improved the situation, still already in 2006 the interest was declining again and/or intermittent. In Portugal, the education/outreach/engagement activities from 2007 up to now, with a great involvement of the teachers, changed the panorama in a spectacular way. In the figure below, it is presented the case for the evolution of the minimal application marks to enrol in a certain graduation course at a Portuguese university.

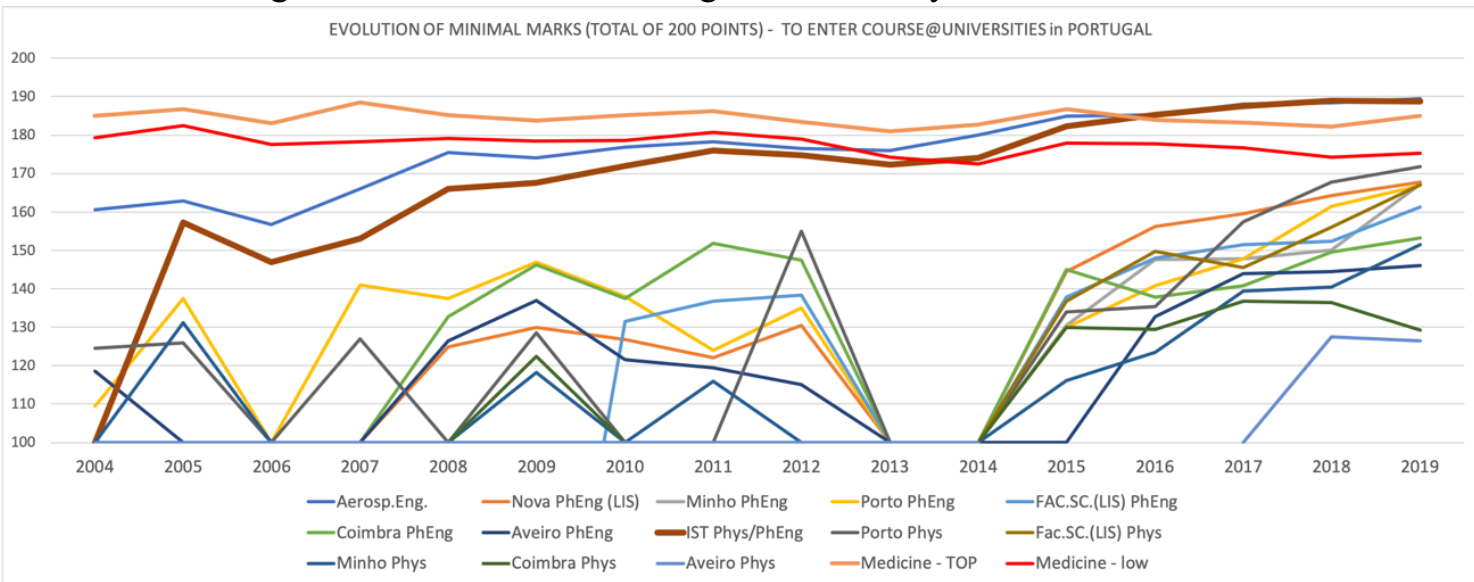

Figure 1. Evolution of minimal application marks to join a graduation course in a portuguese university. Values range from 100 to 200. If the vacancies available are not fulfilled in the first application phase, the minimal mark is set to 100. These numbers are not normalised to the number of vacancies in each course. 
In what follows I present a small account of some of the contributions to this very positive evolution of the interest of students to study Physics/Engineering Physics at universities.

As many countries in the Europe and around the world with similar application process to higher education (after secondary school and typically at a University), Medicine studies were in 2006 at the top of the choices of students, with a fierce competition for places turning into minimal application marks above 175/200. The top course in engineering - Aerospatiale Engineering was not far below Medicine and always held a high interest in the students. But many Physics and Engineering Physics courses were not even filled in the first application phase, calling for action to revert the "disaster" in 2006.

In Portugal we succeeded with the help of the teachers. Realising that the interface between scientists and the students is very often their school teachers, CERN has launched its teachers programmes in 1998, in which during three summer weeks, about 48 high-school teachers from the member states and other places gathered at CERN for an intense training programme. These international programmes happen now twice a year (the "High-School Teachers" at CERN and the "International Teachers Weeks"), for a duration of two weeks. The importance of these teachers programmes is very big, as it updates the teachers with contents from modern physics for many not taught in their graduation time - in the facilities where the actual science is developed. Integrating lectures, visits to the experiments and hands-on activities, allows the teachers to experience the real life of a scientist, while at the same time learning concepts and tools to pass them to their students. And, naturally, engages the scientists - as colleagues or as role models - with the estimated one thousand students that one teacher will encounter in the average 10 years of his/her future career.

But the participation of teachers from one single country, say it, Portugal, is too small ( 1 or 2) for a real change in the teachers engagement in that country. So, when in 2006 CERN proposed the "National Teachers Programmes", Portugal immediately grabbed the idea and started the Portuguese Teachers Programmes in 2007 (in Portuguese). The advantages are enormous:

- Many more opportunities per country, allowing to build a solid network;

- Only one week training, that can be taken from the teachers obligatory yearly training time (instead of three or two weeks of vacations time);

- With all the lectures, visits and activities in (one of) the language(s) spoken in the country, eliminates whatever barriers might exist for teachers to ask questions and participate in the discussions. This is a big advantage, as the subject at hand is already difficult and, making a teacher to express his/her doubts in a foreign language, whatever his/her language skills might be, quickly turns out into teachers being silent;

- Involving Portuguese scientists as speakers, guides, monitors, also provides the teachers with a solid network of scientists that can be invited to schools, collaborate in school activities, be a role model for the students, participate in online sessions and virtual visits, etc.

In 2009 the programme was extended to other Portuguese speaking countries, becoming the CERN's Portuguese Language Teachers Programmes (PTLTP), starting with Brazil and Mozambique, and including teachers from all Portuguese speaking countries in 2011, of whom a photograph is shown in Figure 2. 


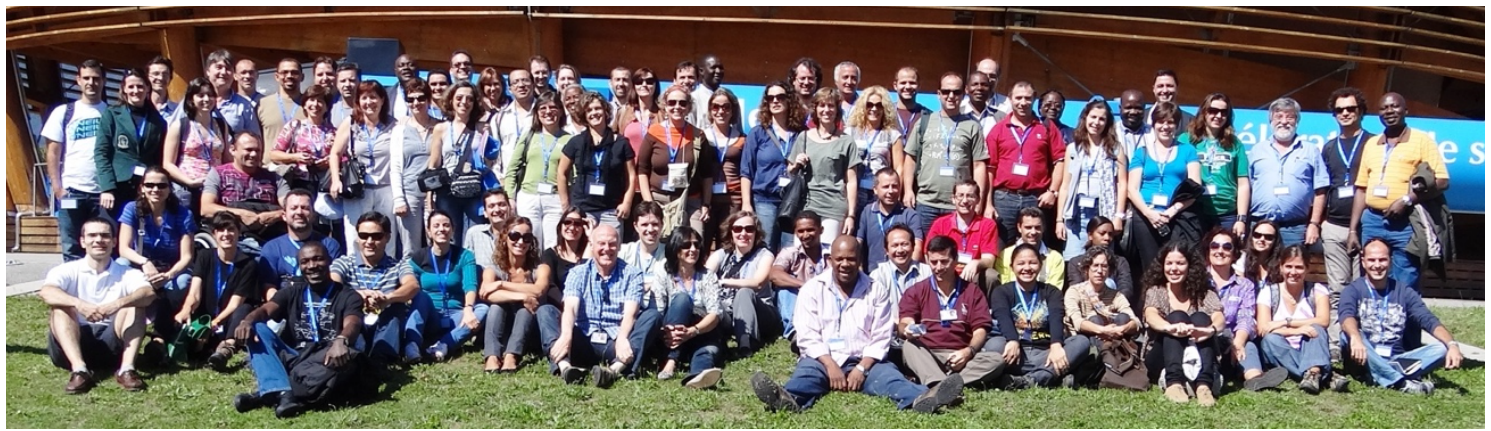

Figure 2. The 73 participants in the CERN's PTLTP2011, with some of the speakers and Coordinators. Participants came from Portugal (41 Pt.), Brazil (20 Br.), Angola (4 Ao.), Mozambique (4 Mz.), Cape Verde (CV), São Tomé e Príncipe (STP), Guinea-Bissau (GB) and East-Timor (TL) (1 each).

The numbers of teachers participating in the CERN's Portuguese Language Teachers Prorammes since its start and up to now are displayed in table 1.

\begin{tabular}{|c|c|c|c|c|c|c|c|c|c|c|c|c|c|c|}
\hline & $\mathbf{2 0 0 7}$ & $\mathbf{2 0 0 8}$ & $\mathbf{2 0 0 9}$ & $\mathbf{2 0 1 0}$ & $\mathbf{2 0 1 1}$ & $\mathbf{2 0 1 2}$ & $\mathbf{2 0 1 3}$ & $\mathbf{2 0 1 4}$ & $\mathbf{2 0 1 5}$ & $\mathbf{2 0 1 6}$ & $\mathbf{2 0 1 7}$ & $\mathbf{2 0 1 8}$ & $\mathbf{2 0 1 9}$ & Total \\
\hline Cand. & 205 & 241 & 191 & 218 & 190 & 250 & 162 & 236 & 187 & 183 & 170 & 181 & 222 & 2063 \\
\hline $1^{s t}$ Cand. & 205 & 169 & 78 & 130 & 98 & 165 & 57 & 121 & 96 & 63 & 40 & 48 & 77 & 1182 \\
\hline \hline Pt. & 43 & 45 & 44 & 45 & 41 & 35 & 34 & 35 & 24 & 22 & 20 & 20 & 20 & $\mathbf{4 2 8}$ \\
\hline Br. & & 1 & 11 & 20 & 20 & 30 & 31 & 30 & 22 & 20 & 20 & 20 & 20 & $\mathbf{2 4 5}$ \\
\hline Mz. & & & 5 & 4 & 4 & 4 & & 2 & 2 & 1 & & & & $\mathbf{2 2}$ \\
\hline TL & & & & & 1 & 3 & 3 & & 2 & & & & & $\mathbf{9}$ \\
\hline STP & & & & 1 & 1 & 1 & 1 & 1 & 1 & 1 & & & & $\mathbf{7}$ \\
\hline Ao. & & & & & 4 & & & & & & & & 2 & $\mathbf{6}$ \\
\hline CV & & & & 1 & 1 & 1 & & 1 & 1 & & & & & $\mathbf{5}$ \\
\hline GB & & & & & 1 & & & & & & & & & $\mathbf{1}$ \\
\hline Total & $\mathbf{4 3}$ & $\mathbf{4 6}$ & $\mathbf{6 0}$ & $\mathbf{7 1}$ & $\mathbf{7 3}$ & $\mathbf{7 4}$ & $\mathbf{6 9}$ & $\mathbf{6 9}$ & $\mathbf{5 2}$ & $\mathbf{4 4}$ & $\mathbf{4 0}$ & $\mathbf{4 0}$ & $\mathbf{4 2}$ & $\mathbf{7 2 3}$ \\
\hline
\end{tabular}

Table 1. Evolution with year of the numbers of participants in the CERN's PTLTP. The top lines give the numbers of portuguese candidates (all candidates and only candidates for the first time).

These programmes have had an immediate impact on the participation of secondary schools students in activities related to particle physics (mostly organized by LIP in Portugal) and to CERN (school visits to CERN and virtual visits, for example), as can be concluded from the evolution of the numbers of participants in the IPPOG's International Masterclasses in Particle Physics and the numbers of schools visiting CERN each year in Figure 3.

In these distributions, the effect of the start of the Portuguese Teachers Programmes in 2007 is notorious, showing the rise of the interest of students that is also correlated with the increase of their interest in choosing physics or engineering physics as their future course in a Portuguese university, as displayed in Figure 1. In particular, one of the Physics courses proposed - the integrated master's degree in physics engineering and Technology at IST (Técnico Lisboa) - overcome the minimal marks to study Medicine in 2014 (lowest marks) and 2016 (highest marks). Maybe more relevant is the fact that all courses in physics/engineering physics and related fields in Portuguese Universities fill up their quotas in the first application phase since 2016.

It also shows clearly that the efforts had a high return and that the outreach activities and engagement of the students have an important impact, so much more when involving the teachers at the secondary schools, as they are the key factor in motivating the students and in linking different generations of students. 


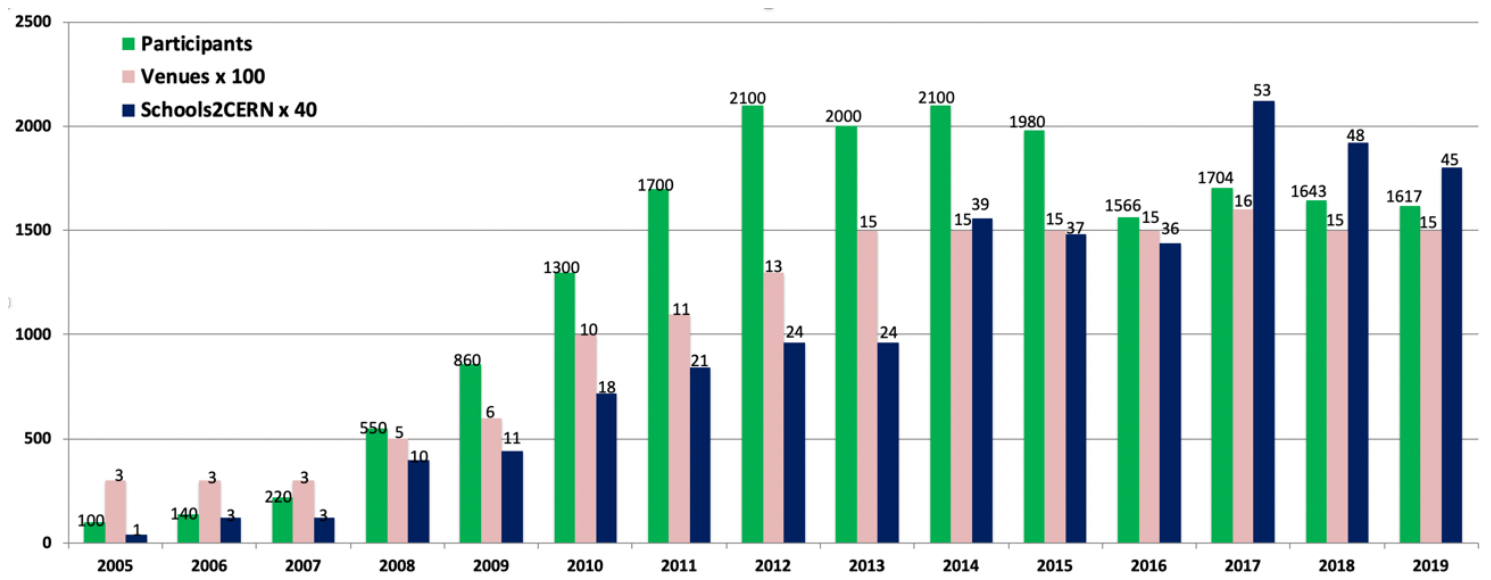

Figure 3. Numbers of portuguese participants in IPPOG's International Masterclasses in Particle Physics and numbers of portuguese schools visits to CERN (above the columns are placed the real numbers).

\section{It's all about Networks and Activities}

Most of the education and outreach activities in particle physics benefit strongly from the existence of networks. Networks exchange ideas and best practices, spread the word, engage with different audiences and enable to reach further.

In 1998, answering to a challenge put forward by then CERN's Director-General, Chris Lewellin-Smith, ECFA and the HEPP board of the EPS launched EPOG - European Particle physics Outreach Group, a committee of representatives from the CERN Member States and from the LHC major experiments to share ideas and best practices related to education and outreach in particle physics. The group met twice a year for the members to report the activities in the country and in 2005 launched the International Masterclasses in Particle Physics - Be a Scientist for a Day... with the Hands-on Particles (IMC for short), which quickly became its flagship activity. The group changed the name into IPPOG - International Particle Physics Outreach Group in 2011 when it incorporated members from non-European countries (for example, United States, South Africa, Israel, Brazil, etc), and became a formal international collaboration based on a Memorandum of Understanding in 2016 (IPPOG presentation by S.Goldfarb at this Conference).

IPPOG - www.ippog.org - is a collaboration of scientists, with experience in education and outreach, professional communicators, experts in communication and outreach, researchers at universities and teachers at high-schools. Each member of the collaboration represents a country, an experiment or an international laboratory (national laboratories relevant in education and outreach in particle physics can be associated members of the collaboration). At the time of the conference, the collaboration comprised 26 countries, 6 experiments and 1 international laboratory (list of members at ippog.org/members). In addition to the member representatives there are the forum members that contribute significantly to the IPPOG activities, carry out the IPPOG activities in their countries and institutions, share the ideas and the results in the bi-annual meetings, or propose new activities, tools, methods to engage the students and the public at all ages.

IPPOG launched in 2017 the Global Cosmics network, a federation of projects involving Cosmic Rays, to coordinate global activities related to Cosmic Rays (globalcosmics.org). It is responsible for the International Cosmic Day (ICD - icd.desy.de) and collaborates in the organization/promotion of the International Muon Week run by quarknet (quarknet.org). It also maintains the website cosmicatweb.desy.de, that lists several projects with cosmic rays data available online. A presentation of Global Cosmics (N.Arnaud) was made at this Conference.

The flagship activity of IPPOG, the International Masterclasses in Particle Physics, was also featured at this Conference in a report by U.Bilow and K.Cecire, in which they present the 
structure and main progresses in this programme, as well as the paths for scientists to get involved. It is a programme running for nearly 7 weeks in the spring, in which secondary schools students are invited to spend a day at a local university (or research institute), experiencing how it is like to "Be a Scientist for a day... with the hands-on particles". Launched in 2005, follows the initial programme started in 1997 in the UK [2], but adding the final video-conference linking moderators at large laboratories (CERN, Fermilab, KEK, GSI, TRIUMF) and the students that have participated in that day at up to 5 institutes/places. The day starts with introductory talks about Particle Physics, Accelerators, Detectors, Technologies, etc, followed by lunch. Then, the students analyse real data from experiments at CERN and other places, make their own measurements and take their own conclusions. Then they join a videoconference in which the moderators discuss the results with the students, the advantages of statistics and collaboration, and answer questions posed by the students.

The video-conference concludes with a fun quiz, after one hour of enthusiastic interactions (sometimes up to two hours). Today participate in each yearly edition about 60 countries, 220 institutes and 14000 students. In 2020, due to the

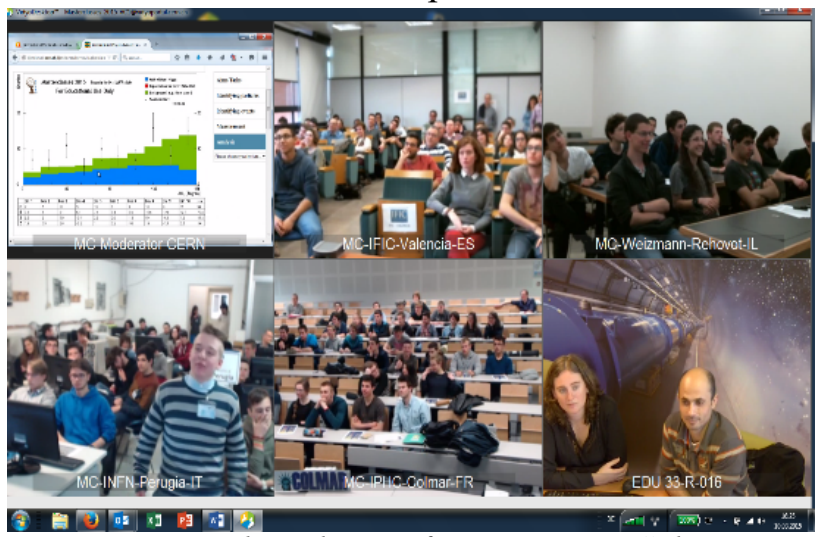

Figure 4. The video-conference in a IMC day pandemic situation, only about $25 \%$ of the sessions took place. To compensate for this, a more simple online based activity - BAMC: Big Analysis of Muons in CMS - was realized with the participation of 700 students.

In this conference were also presented the new measurements ("Darkside" based at Gran Sasso, and "Microboone" in preparation), in addition to updates in the measurements already present in a regular way (from the four LHC experiments, MINERvA, BELLE-II and Particle Therapy).

The other networks presented at this conference were OCRA (Outreach Cosmic Rays Activities - www.infn.it/OCRA/ - by C.Aramo), a new outreach network in the field of Gravitation and Gravitational Waves with members from the LIGO and VIRGO/EGO Collaborations/Observatories (iGrav - www.igrav.org - by N.Arnaud), and the impressive "Netzwerk Teilchenwelt" in Germany (U.Bilow). standing out in the latter the multi-step approach for high-school students (3000 students participate in a IMC-like masterclass, about 250 engage actively in a detector project, typically 60 participate in immersive workshops at CERN, and finally 15 carry on further their own research projects).

\section{It's all about You}

In this section I discuss briefly some highlights of the Education and Outreach track of this Conference. The most important highlight is the track itself, already a large success since its introduction in ICHEP2012 (by invitation only) and regularly accepting contributions from ICHEP2014 onwards. But in this Conference, the success measures already by the large number of oral presentations, summing up to 33 talks in its 4 parallel sessions, covering 7 topical areas in education and outreach. Having discussed networks and its flagship activities in the previous section, here I discuss briefly the contributions in the other 6 topics. The division of the contributions in these topics was entirely my choice.

As public engagement activities, there were reports about the CERN Open Days in September 2019, two heavy days in which CERN opened its doors to 45000 people per day, and the experiments at CERN prepared activities for the visitors and organized tours to the experimental caverns. The visit to ALICE (D. Hatzifotiadou) included a tour to the LHC accelerator for a total time underground of 28 minutes (give or take a millisecond). Both ALICE, 
ATLAS (A. Sfyrla) and CMS (M.Lapka) presented highly engaging activities proposed on-site at the surface level, for people waiting to go underground or just passing by the site, including the "Learn by Playing" activity presented by S.Melhase at the ATLAS site. K.Leney presented his proton/particle cookies engaging with the stomachs of CERN visitors in "A Taste of Physics". Open days were also reported from Trento Proton Therapy Centre (B. Di Ruzza) and A.Gritsan reported on the engagement activities at the LHC stand of the Science Festival at the Washington D.C.. C.Potter presented the science engagement activities carried out at several music festivals (with WOMAD standing out as a huge success, in which a large fraction of the tens of thousands of participants queued to visit the Science Pavillion between concerts), allowing to engage with different audiences. Finally there were important developments in the

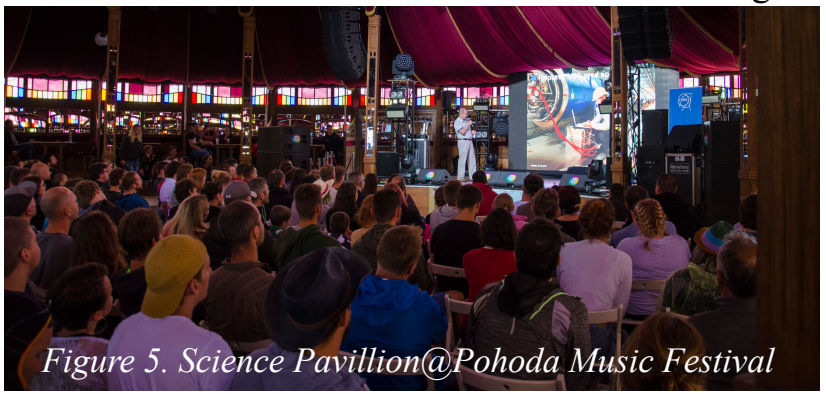
connections between arts and science, in workshops and shows reported by K.Nikolopoulos, and in a report about the Origin's Exhibition "Meet the Universe" in Oman from M.Hoch.

In Education Programmes there were presentations about an internship of two weeks in the summer for university Italian students (S. Donati), a research internship of one semester for undergraduate students at CERN (from the U.Michigan, presented by S.Goldfarb), and two presentations about the usage and availability of open data from ATLAS (K.Shaw) and CMS (P. Veteli) experiments.

The case for Digital Tools and WEB Presence was well represented in reports by M.Evans on WEB Development and port of the ATLAS Website to Drupal 8, on the development of games and gamelike applications (report of an adventure/digital visit to CMS by P.van Hove and on a game involving number guessing within ATLAS by P. Traczyk), mixed reality applications by M. Phirstkhalava and augmented reality using the mobile phone ("tracer.web.cern.ch") by L.Khelashvili, and virtual reality developed for 3D hardware at science fairs (by A.Peixoto). Finally we had two important reports on Virtual Visits at ATLAS (B.Carlson) and at CMS (C.Uribe Estrada), that show the increasing vitality of these offers, enabling students and general public from all over the world to visit the ATLAS or the CMS experiments.

On Social Media we had a report about good practices on the use of the different social media platforms by V.Scotti and a report about statistics of ATLAS presence in social media by K.Anthony, that showcase the importance and strength on the use of social media to expand the reach and the engagement of the [digital] population.

Two Studies were presented related to surveys. One regarding important points in spreading the interests of students in particle physics (S.Zoechling) and a very interesting and exhaustive study on Career Trajectories after CERN (J.Niedziela), which presents very useful data that we can use when motivating students to study physics and do a Ph.D. at CERN or related subjects (some examples in Figure 6).

And on a more Philosophy of science tone we had a beautiful presentation and discussion about Beauty in Physics (and possible induced prejudices) by I.Melo.
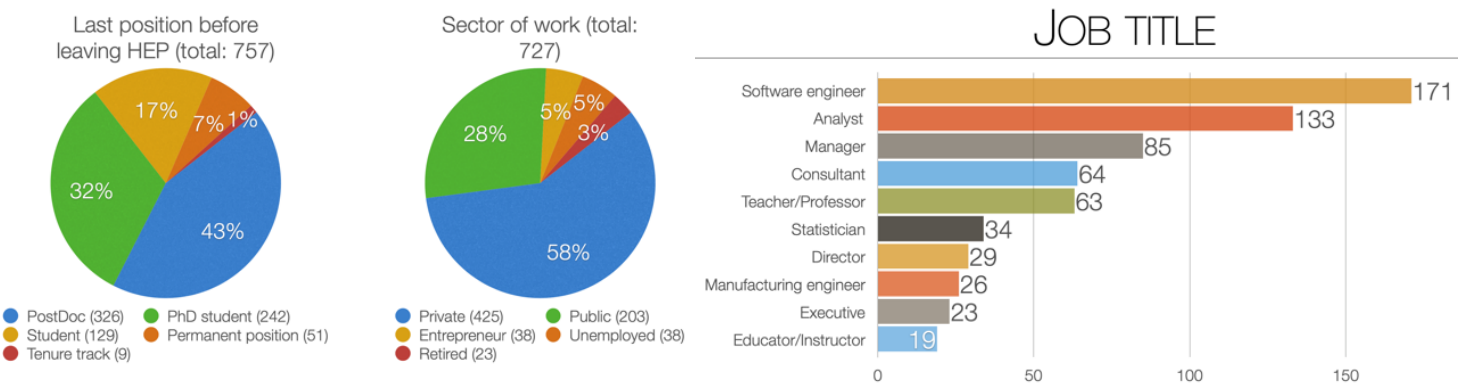

Figure 6. Career Trajectories after CERN 


\section{Conclusions}

The present times, especially in the aftermath of the approval by the CERN Council last June of the Update of the European Strategy for Particle Physics, poses big challenges for education and outreach, if we want to be successful in carrying out our projects and maintain our highly productive field of research thriving in the near- and long-term future.

Among these challenges stand out:

- The scale of [new] projects (timespan, cost, size, ...);

- Wide[r] recognition of the value of [fundamental] Science in the Society;

- Increase the reach and broadness of our activities (research as well as education and outreach) - geographically, diversity, inclusion, age;

- Train the trainers - Teachers, Educators, Tutors, Motivators; and

- Introduction of high energy and particle physics related contents in the school curricula - either adiabatically or with a swift phase transition (a.k.a. revolution)

Education and Outreach are more important than ever! And are crucial to meet and overcome the above challenges, which are all strongly correlated.

Moreover, as stated in the ESPPU'2020, we need to maintain our relevance in society and work on how the public perceives the scientific knowledge and the scientific process.

So, in this conference it has become clear that

- good Education, Communication and Outreach are essential pillars for the development of high energy and particle physics;

- many scientists, at different levels of commitment and along with experts in outreach and communication, are already carrying outwards the excitement of the field with many activities and engaging the future generation of scientists.

But we all need You! Our field desperately needs Your support and contribution,

- as actively being an ambassador of the field,

- as speaker/moderator/motivator/organiser/educator/tutor/musician,

- as evaluator of projects/career assessment/applications/positions,

- and/or helping to create/improve the conditions for a better EducationCommunication-Outreach in high energy and particle physics.

Thanks for your attention and to all the people promoting and collaborating in all the activities described in this report, in particular those that turned the parallel sessions in the Education and Outreach so exciting and successful.

\section{O_TREACH is not complete without $\mathbf{U}$}

\section{References}

[1] M.Bardeen, H.P.Beck, U.Bilow, K.Cecire, M.Kobel, F.Ould-Saada, International Masterclasses in the LHC era, CERN Courier 54, n.5 (2014) 37.

[2] R. Barlow, International Masterclasses in the LHC era, CERN Courier 54, n.5 (2014) 37. 